\title{
Resonantly Enhanced Second and Third Harmonic Generation in Microfibre Loop Resonators
}

\author{
T. Lee ${ }^{1 *}$, N. G. R. Broderick ${ }^{2}$, R. Ismaeel ${ }^{1}$, M. A. Gouveia ${ }^{1}$, \\ G. Brambilla ${ }^{1}$ \\ 1. Optoelectronics Research Centre, University of Southampton, Southampton, SO17 1BJ, \\ United Kingdom \\ 2. Physics Department, University of Auckland, Private Bag 92019, Auckland, New Zealand \\ *Corresponding author:tl305@orc.soton.ac.uk
}

Due to their strong modal confinement, optical microfibres (OMs) provide an excellent opportunity for studying nonlinear effects and in particular intermodally phase matched second (SHG) and third (THG) harmonic generation [1-3] with predicted efficiencies up to $10^{-1}$ over several $\mathrm{cm}$ in an ideal uniform silica OM for both cases. Experimentally however, fabricating the required phase matching diameter uniformly over such lengths remains challenging. A simple solution is to reconfigure the $\mathrm{OM}$ as a resonator, such that pump recirculation near resonance increases conversion without the need for higher power sources or OM lengths greater than a few $\mathrm{mm}$. Here, we theoretically study surface-SHG and THG in OM loop resonators [4] by numerically solving the underlying coupled mode equations to determine the dependence of the enhancement on the resonance properties.
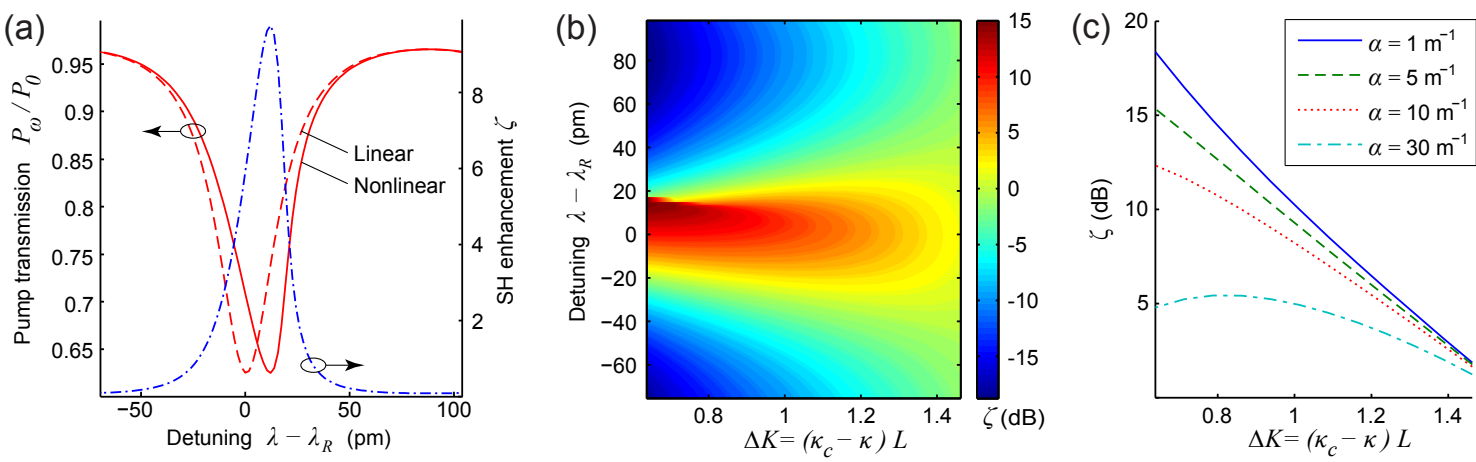

Fig. 1. (a) The linear (dash) and nonlinear (solid) pump transmission spectrum of a $4 \mathrm{~mm}$ diameter loop resonator when detuned near a resonance at $\lambda=1.55 \mu \mathrm{m}$, and the corresponding second harmonic enhancement factor $\zeta$ (dash-dot). $P_{0}=100 \mathrm{~W}$, loss $\alpha=5 \mathrm{~m}^{-1}$ and proximity to critical coupling is $\Delta K=1$. (b) The second harmonic enhancement as a function of proximity to critical coupling and detuning from resonance, where $\kappa, \kappa_{c}$ and $L$ are the coupling, critical coupling and length of the loop coupling region, respectively. (c) The dependency of the maximum enhancement on the microfibre loss $\alpha$.

For a resonance near $\lambda \approx 1.55 \mu \mathrm{m}$, Fig. 1(a) shows the linear transmission of a typical $4 \mathrm{~mm}$ diameter loop resonator and the nonlinear transmission at a pump power of $P_{0}=100 \mathrm{~W}$. SHG is assumed over a $1 \mathrm{~mm}$ length within the loop. On resonance, the second harmonic efficiency is enhanced by a factor of $\zeta=10$ times greater than the efficiency from an equivalent $1 \mathrm{~mm}$ long straight $\mathrm{OM}\left(\eta_{\mathrm{OM}}=1 \times 10^{-5}\right)$. The enhancement spectrum appears asymmetric and redshifted from the linear resonance spectrum (as reflected in the nonlinear pump spectrum) due to nonlinear phase modulations since the internal pump power is over 3 times greater than the input power; an effect which can introduce bistability at higher pump powers. Fig. 1(b) confirms that nearer to critical coupling (smaller $\Delta K$ ), the stronger resonance and greater pump recirculation results in a higher enhancement of $\zeta=35$ $(15 \mathrm{~dB})$ at $\Delta K=0.7$. However, the higher $\mathrm{Q}$ resonance reduces the bandwidth to $20 \mathrm{pm}$, or $12 \%$ of the free spectral range (FSR), whereas a weaker resonance at $\Delta K=1.4$ would offer a wider bandwidth of $26 \%$ of the FSR at the cost of a much reduced enhancement $(\zeta=2)$. Practically, the OM may be subject to surface dust and moisture contamination, but the enhancement remains significant even for a loss of $\alpha=10 \mathrm{~m}^{-1}$ as seen in Fig. 1(c). Whereas the SHG efficiency increases quadratically with pump power, for THG the dependence is cubic which leads to much greater resonant enhancements. Indeed, for a $3 \mathrm{~mm}$ long loop, THG efficiencies up to several percent can be attained, with $\zeta=640\left(\Delta K=0.7\right.$ and $P_{0}=100 \mathrm{~W}$ as before), and the peak recirculating power of $860 \mathrm{~W}$ would be well tolerated by the loop if using nanosecond pulses. Finally, it should be noted that the conversion can be increased yet further when the harmonic wavelength is co-resonant with the pump.

\section{References}

[1] V. Grubsky and A. Savchenko, "Glass micro-fibers for efficient third harmonic generation,” Opt. Express 13, 6798-6806 (2005).

[2] V. Grubsky and J. Feinberg, "Phase-matched third-harmonic UV generation using low-order modes in a glass micro-fiber," Opt. Commun. 274, 447-450 (2007).

[3] J. Lægsgaard, "Theory of surface second-harmonic generation in silica nanowires," J. Opt. Soc. Am. B 27, 1317-1324 (2010).

[4] M. Sumetsky, Y. Dulashko, J. Fini, A. Hale, and D. DiGiovanni, "The microfiber loop resonator: Theory, experiment, and application," J. Lightwave Tech. 24, 242-250 (2006). 